\title{
Spin Accumulation in a Quantum Wire with Rashba Spin-Orbit Coupling
}

\author{
Xi Fu, ${ }^{1,2}$ Wenhu Liao, ${ }^{1}$ and Guanghui Zhou ${ }^{1}$ \\ ${ }^{1}$ Department of Physics, Hunan Normal University, Changsha, Hunan 410081, China \\ ${ }^{2}$ Department of Electronics and Physics, Hunan College of Science and Engineering, Yongzhou, Hunan 425100, China
}

Correspondence should be addressed to Guanghui Zhou, ghzhou@hunnu.edu.cn

Received 22 September 2008; Revised 9 December 2008; Accepted 17 December 2008

Recommended by Yuri Galperin

We investigate theoretically the spin accumulation in a Rashba spin-orbit coupling quantum wire. Using the scattering matrix approach within the effective free-electron approximation, we have demonstrated the three components of spin polarization. It is found that by a few numerical examples, the two peaks for the out-of-plane spin accumulation $\left\langle S_{z}\right\rangle$ shift to the edges of quantum wire with the increase of propagation modes. The period of intrinsic oscillations $\left\langle S_{x / y}\right\rangle$ inversely proportions to the Rashba SOC strength. This effect may be used to differentiate the intrinsic spin accumulation from the extrinsic one.

Copyright (C) $2008 \mathrm{Xi} \mathrm{Fu}$ et al. This is an open access article distributed under the Creative Commons Attribution License, which permits unrestricted use, distribution, and reproduction in any medium, provided the original work is properly cited.

Spintronics is a rapidly developing field of research in recent years because spin-based devices are believed to be the new generation of electronic devices [1-3]. Among the research area of spintronics, the spin-orbit coupling (SOC) creates another way to manipulate spins by means of an electric field [3], which has great potential applications in the future. The Rashba spin-orbit coupling (RSOC) effect is found to be very pronounced in some semiconductor layer (e.g., InAs or InGaAs) and its strength can be controlled by gate voltage [4].

The spin polarization in two-dimensional electron gas (2DEG) systems with SOC has been attracting much attention [5-9]. Recently, the experimental observations [10, 11] of spin Hall effect (SHE) in 2DEG with SOC have given evidence of the possibility to control electron spins by an electric field. Wang and Sheng [12] have investigated the current induced local spin polarization due to weak RSOC in a narrow strip. Furthermore, Nikolić et al. [13] have demonstrated that the flow of a longitudinal unpolarized current through a ballistic 2DEG with RSOC will induce a nonequilibrium spin accumulation along the lateral edges. More recently, the out-of-plane spin Hall accumulation effect has been observed in a Rashba bar [14]. Using the nonequilibrium Green's function approach, Wang et al. [15] have studied the intrinsic oscillation of spin accumulation induced by RSOC in a quantum wire (QW) system. However, the work in $[12,14,15]$ has only considered the hard-wall potential confined Rashba QWs.

In this paper, using the scattering matrix method within the effective free-electron approximation, we study theoretically the spin accumulation in a parabolic potential confined Rashba QW connected to two normal leads (without SOC) nonadiabatically, and the results are discussed and compared with the previous works [12-15]. The system under consideration is a wide-narrow-wide (WNW) geometry of structure, and the number of propagation modes in the narrow QW and wide leads is controlled by the different strength of parabolic confining potentials. The transverse direction corresponds to $x$-axis and the longitudinal direction to $y$-axis. Therefore, the effective mass single-electron Hamiltonian in the QW is

$$
H=H_{0}+H_{s o}
$$

where

$$
H_{0}=-\frac{\hbar^{2}}{2 m^{*}}\left(\frac{\partial^{2}}{\partial x^{2}}+\frac{\partial^{2}}{\partial y^{2}}\right)+V(x)
$$

is the uncoupled QW Hamiltonian while the RSOC Hamiltonian $[14,15]$ reads

$$
H_{s o}=i \alpha_{R}\left(\sigma_{y} \frac{\partial}{\partial x}-\sigma_{x} \frac{\partial}{\partial y}\right) .
$$


In the above Hamiltonians, $V(x)=m^{*} \omega^{2} x^{2} / 2$ is the lateral confining potential with $m^{*}$, and $\omega$ the effective electron mass and oscillator frequency, while $\alpha_{R}$ denotes the Rashba strength, and $\sigma_{x / y}$ the $x / y$ component of pauli matrix.

Since the Hamiltonian in the leads has no RSOC term, we assume that an unpolarized electron wave is injected from the left lead into the QW. Due to the translation invariance in the longitudinal $y$-direction, the electron wave function in the leads can be decomposed into two components of a plane wave and a transversally confined wave function for the $\mathrm{N}$ th mode

$$
\phi_{N \uparrow \downarrow}(\alpha x)=\frac{e^{-(\alpha x)^{2} / 2}}{\sqrt{2^{N} N ! \sqrt{\pi}}} H_{N}(\alpha x) \quad(N=1,2, \ldots),
$$

where $\uparrow(\downarrow)$ represents spin-up (-down) state, $\alpha=\left(m^{*} \omega / \hbar\right)^{1 / 2}$, and $H_{N}(\alpha x)$ is the Hermite polynomial. Since no RSOC in the leads, the total electron energy for spin-up or -down electron is same, $E=\hbar^{2} K_{N}^{2} / 2 m^{*}+\varepsilon_{N}$, where the lateral sublevel $\varepsilon_{N}=(N+1 / 2) \hbar \omega$ and the longitudinal wavevector $K_{N}=\sqrt{2 m^{*}\left(E-\varepsilon_{N}\right)} / \hbar$.

The wave function for the weak Rashba QW is a twocomponents spinor $\psi(x, y)=\left\{\psi_{\uparrow}(x, y) \psi_{\downarrow}(x, y)\right\}$ and $\psi_{\uparrow \downarrow}(x, y)$ can be also decomposed into two components for spinup $(\uparrow)$ and spin-down $(\downarrow)$ electron, respectively. The corresponding transverse ( $x$ direction) wave functions in the perturbation regime [16] can be solved as

$$
\begin{aligned}
& \varphi_{n \uparrow \downarrow}(\alpha x) \\
& \qquad=\frac{e^{-(\alpha x)^{2} / 2}}{\sqrt{2^{n} n ! \sqrt{\pi}}}\left\{H_{n}(\alpha x) \pm \frac{l_{\omega}}{l_{\alpha_{R}}}\left[n H_{n-1}(\alpha x)+\frac{1}{2} H_{n+1}(\alpha x)\right]\right\},
\end{aligned}
$$

where $l_{\alpha_{R}}=\hbar^{2} /\left(2 m^{*} \alpha_{R}\right)$ and $l_{\omega}=1 / \alpha$ the character length related to the oscillator frequency, and the sign " + /-" corresponding to “ $\uparrow / \downarrow$ ” state. The electron energy in the QW is $E_{\uparrow \downarrow}=\hbar^{2} k_{n \uparrow \downarrow}^{2} / 2 m^{*}+\varepsilon_{n \uparrow \downarrow}$, where the lateral sublevel of the $n$th mode is $\varepsilon_{n \uparrow}=(n+1 / 2) \hbar \omega+\alpha_{R} k_{n \uparrow}$ and $\varepsilon_{n \downarrow}=$ $(n+1 / 2) \hbar \omega-\alpha_{R} k_{n \downarrow}$, respectively. Therefore, the longitudinal wavevectors can be expressed as

$$
\begin{aligned}
& k_{n \dagger}^{ \pm}\left(E_{\uparrow}\right)=\frac{m^{*}}{\hbar^{2}}\left[-\alpha_{R} \pm \sqrt{\alpha_{R}^{2}+2 \hbar^{2} \frac{E_{\uparrow}-(n+1 / 2) \hbar \omega}{m^{*}}}\right], \\
& k_{n \downarrow}^{ \pm}\left(E_{\downarrow}\right)=\frac{m^{*}}{\hbar^{2}}\left[\alpha_{R} \pm \sqrt{\alpha_{R}^{2}+2 \hbar^{2} \frac{E_{\downarrow}-(n+1 / 2) \hbar \omega}{m^{*}}}\right]
\end{aligned}
$$

for spin-up and -down electron, respectively.

In order to calculate the scattering matrix separately for spin-up and -down electron, one needs to match the wave functions at the two interfaces of wire-lead connection. For a symmetric system, one can only calculate the scattering matrix for one interface. The total scattering matrix for the system can be obtained by the symmetry property of the system [17]. Here, we first consider the left interface at $y=0$ as if without the right interface $(y=L)$ for electron incident from left to right. Because of the degeneracy, in the left lead $(y<0)$, the wave functions for spin-up and -down electron can be written as

$$
\psi_{\uparrow \downarrow}(x, y)=\phi_{N \uparrow \downarrow}(\alpha x) e^{i K_{N} y}+\sum_{N^{\prime}} r_{\uparrow \downarrow N^{\prime} N} \phi_{N^{\prime} \uparrow \downarrow}(\alpha x) e^{-i K_{N^{\prime}} y},
$$

where $r_{\uparrow \downarrow N^{\prime} N}$ are the coefficients of incident mode $N$ reflecting to mode $N^{\prime}$. In the QW $(y>0)$, the wave functions for spin-up and -down electron are nondegenerate

$$
\psi_{\uparrow \downarrow}(x, y)=\sum_{n} t_{\uparrow \downarrow n N}^{\prime} \varphi_{n \uparrow \downarrow}(\alpha x) e^{i k_{n \downarrow \downarrow}^{+} y}
$$

where $\varphi_{n \uparrow \downarrow}$ are given in (5) and $t_{\uparrow \downarrow n N}^{\prime}$ the coefficients of incident mode $N$, transmitting to mode $n$. The continuity of the wave functions at left interface $y=0$ gives

$$
\phi_{N \uparrow \downarrow}(\alpha x)+\sum_{N^{\prime}} r_{\uparrow \downarrow N^{\prime} N} \phi_{N^{\prime} \uparrow}(\alpha x)=\sum_{n} t_{\uparrow \downarrow n N}^{\prime} \varphi_{n \uparrow \downarrow}(\alpha x) .
$$

The continuity of the derivative of the wave functions may not hold due to the existence of RSOC [16]. However, the current conservation for the system still holds $[18,19]$ :

$$
\begin{gathered}
{\left[\begin{array}{cc}
-i \frac{\hbar}{m^{*}} \frac{\partial}{\partial y} & 0 \\
0 & -i \frac{\hbar}{m^{*}} \frac{\partial}{\partial y}
\end{array}\right]\left[\begin{array}{l}
\psi_{\uparrow}(x, y<0) \\
\psi_{\downarrow}(x, y<0)
\end{array}\right]} \\
=\left[\begin{array}{cc}
-i \frac{\hbar}{m^{*}} \frac{\partial}{\partial y} & \frac{\alpha_{R}}{\hbar} \\
\frac{\alpha_{R}}{\hbar} & -i \frac{\hbar}{m^{*}} \frac{\partial}{\partial y}
\end{array}\right]\left[\begin{array}{l}
\psi_{\uparrow}(x, y \geq 0) \\
\psi_{\downarrow}(x, y \geq 0)
\end{array}\right],
\end{gathered}
$$

hence we can obtain

$$
\begin{aligned}
& K_{N} \phi_{N \uparrow \downarrow}(\alpha x)-\sum_{N^{\prime}} r_{\uparrow \downarrow N^{\prime} N} K_{N^{\prime}} \phi_{N^{\prime} \uparrow}(\alpha x) \\
& =\sum_{n} t_{\uparrow \downarrow n N}^{\prime}\left[k_{n \uparrow \downarrow}^{+} \varphi_{n \uparrow \downarrow}(\alpha x)+\varphi_{n \downarrow \uparrow}(\alpha x) / 2 W\right],
\end{aligned}
$$

where $W=l_{\alpha_{R}}=\hbar^{2} /\left(2 m^{*} \alpha_{R}\right)$ is the effective width of the QW. Multiplying $\phi_{N^{\prime} \uparrow \downarrow}$ and $\varphi_{n^{\prime} \uparrow \downarrow}$ with (9) and (11), respectively, and integrating over the range of $-W / 2<x<W$, one obtains the matrices of reflection $r_{\uparrow \downarrow}$ and transmission $t_{\uparrow \downarrow}^{\prime}$ at the $y=0$ interface as

$$
r_{\uparrow \downarrow}=\lambda_{\uparrow \downarrow} t_{\uparrow \downarrow}^{\prime}-1
$$

with

$$
\begin{aligned}
t_{\uparrow}^{\prime}= & {\left[\chi_{\uparrow}^{-1}\left(\lambda_{\uparrow}^{T} K \lambda_{\uparrow}+p_{\uparrow} k_{\uparrow}^{+}\right)-\left(\lambda_{\downarrow}^{T} K \lambda_{\downarrow}+p_{\downarrow} k_{\downarrow}^{+}\right)^{-1} \chi_{\downarrow}\right]^{-1} } \\
& \times\left[2 \chi_{\uparrow}^{-1} \lambda_{\uparrow}^{T} K-2\left(\lambda_{\downarrow}^{T} K \lambda_{\downarrow}+p_{\downarrow} k_{\downarrow}^{+}\right)^{-1} \lambda_{\downarrow}^{T} K\right], \\
t_{\downarrow}^{\prime}= & {\left[\chi_{\downarrow}^{-1}\left(\lambda_{\downarrow}^{T} K \lambda_{\downarrow}+p_{\downarrow} k_{\downarrow}^{+}\right)-\left(\lambda_{\uparrow}^{T} K \lambda_{\uparrow}+p_{\uparrow} k_{\uparrow}^{+}\right)^{-1} \chi_{\uparrow}\right]^{-1} } \\
& \times\left[2 \chi_{\downarrow}^{-1} \lambda_{\downarrow}^{T} K-2\left(\lambda_{\uparrow}^{T} K \lambda_{\uparrow}+p_{\uparrow} k_{\uparrow}^{+}\right)^{-1} \lambda_{\uparrow}^{T} K\right],
\end{aligned}
$$




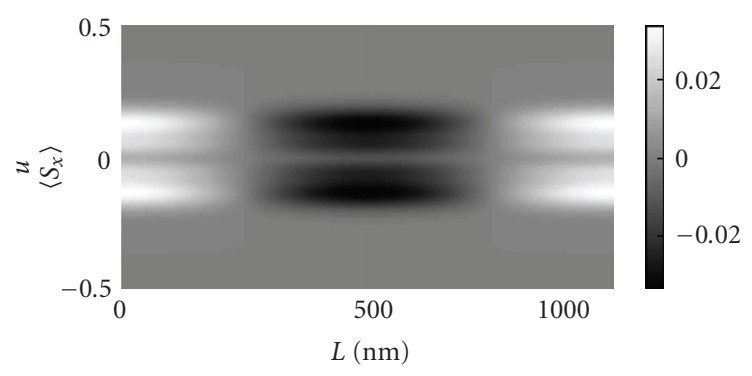

(a)

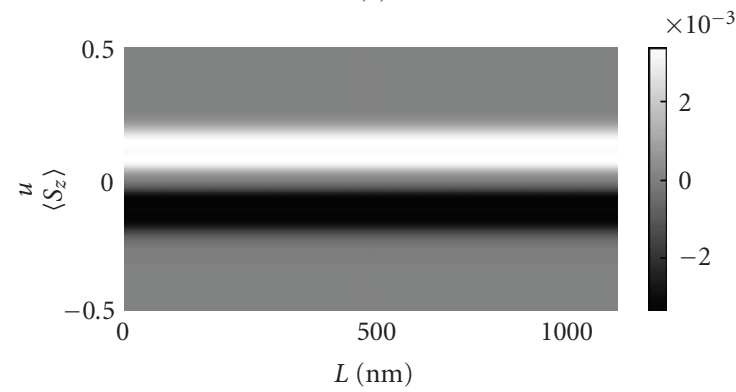

(c)

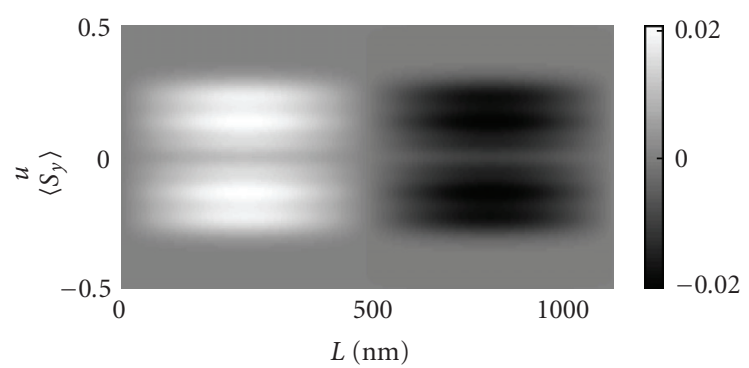

(e)

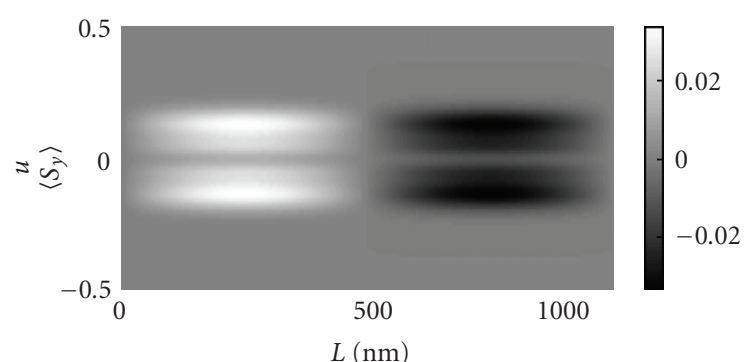

(b)

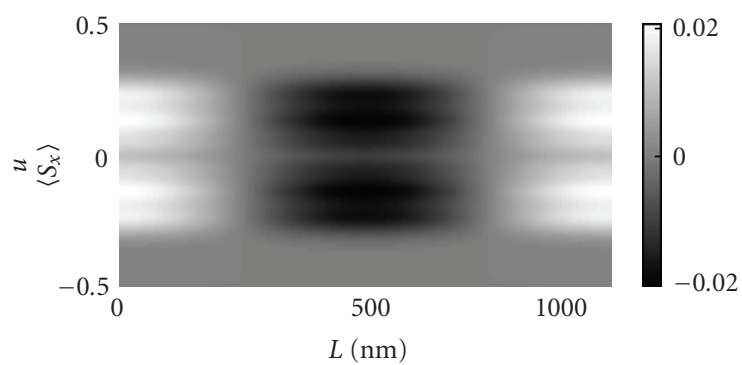

(d)

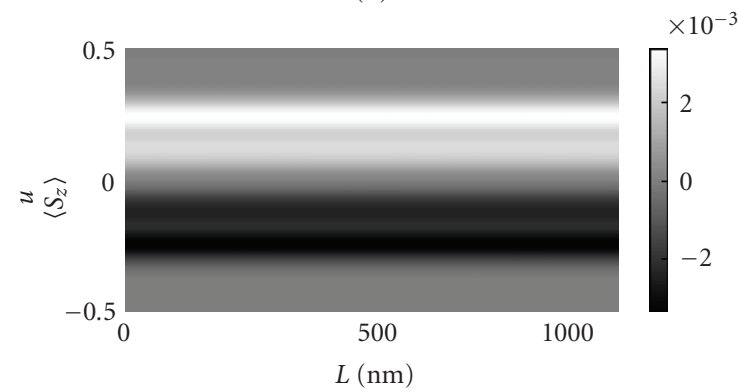

(f)

FIGURE 1: The 2D contour of spin polarization components $\left\langle S_{i}\right\rangle(i=x, y, z)$ as functions of transverse position $u$ and length $L$ for $n=3$ ((a), (b), (c)) and $n=5((\mathrm{~d}),(\mathrm{e}),(\mathrm{f}))$ propagation modes QW with $\alpha_{R}=0.5 \times 10^{-11} \mathrm{eVm}$.

where the corresponding matrices elements are as follows $p_{n n^{\prime} \uparrow(\downarrow)}=\int_{-W / 2}^{W / 2} \varphi_{n \uparrow(\downarrow)}(\alpha x) \varphi_{n^{\prime} \uparrow(\downarrow)}(\alpha x) d x, \chi_{n n^{\prime} \uparrow(\downarrow)}=\left(m^{*} \alpha_{R}\right) / \hbar^{2}$ $\times \int_{-W / 2}^{W / 2} \varphi_{n \uparrow(\downarrow)}(\alpha x) \varphi_{n^{\prime} \downarrow(\uparrow)}(\alpha x) d x, \lambda_{N n \uparrow(\downarrow)}=\int_{-W / 2}^{W / 2} \phi_{N \uparrow(\downarrow)}(\alpha x)$ $\times \varphi_{n \dagger(!)}(\alpha x) d x, k_{n n^{\prime} \dagger(\downarrow)}=k_{n \uparrow(!)} \delta_{n n^{\prime}}$, and $K_{N N^{\prime}}=K_{N} \delta_{N N^{\prime}}$, respectively. Furthermore, an analogous calculation for an incident wave from the right side to the left one at $y=$ 0 interfacegives matrices $r_{\uparrow \downarrow}^{\prime}$ and $t_{\uparrow \downarrow}$. Thus, the scattering matrix $S_{\uparrow \downarrow}^{(1)}$ at $y=0$ interface can be expressed as

$$
S_{\uparrow \downarrow}^{(1)}=\left[\begin{array}{cc}
\tilde{r}_{N N^{\prime} \downarrow \downarrow} & \tilde{t}_{N n \uparrow \downarrow} \\
\tilde{t}_{n N \uparrow \downarrow}^{\prime} & \tilde{r}_{n n^{\prime} \uparrow \downarrow}^{\prime}
\end{array}\right],
$$

where the normalized wave amplitudes $\widetilde{t}_{n N \uparrow \downarrow}^{\prime}=\left(k_{n \uparrow \downarrow}^{+} / K_{N}\right)^{1 / 2} \times$ $t_{n N \uparrow \downarrow}^{\prime}, \tilde{t}_{N n \uparrow \downarrow}=\left(K_{N} / k_{n \uparrow \downarrow}^{-}\right)^{1 / 2} t_{N n \uparrow \downarrow}, \tilde{r}_{N N^{\prime} \uparrow \downarrow}=\left(K_{N} / K_{N^{\prime}}\right)^{1 / 2} r_{N N^{\prime} \uparrow \downarrow}$, and $\widetilde{r}_{n n^{\prime} \uparrow \downarrow}^{\prime}=\left(k_{n \uparrow \downarrow}^{-} / k_{n \uparrow \downarrow}^{+}\right)^{1 / 2} r_{n n^{\prime} \uparrow \downarrow}^{\prime}$. Due to the symmetry of the system, [17] the scattering matrix $S_{\uparrow \downarrow}^{(2)}$ at $y=L$ interface is

$$
S_{\uparrow \downarrow}^{(2)}=\left[\begin{array}{ll}
\widetilde{r}_{n n^{\prime} \downarrow \downarrow}^{\prime} & \tilde{t}_{n N \uparrow \downarrow}^{\prime} \\
\tilde{t}_{N n \uparrow \downarrow} & \tilde{r}_{N N^{\prime} \downarrow \downarrow}
\end{array}\right] \text {. }
$$

Therefore, we can obtain the total wave functions in the QW as

$$
\begin{aligned}
\Psi(x, y) & \\
= & \left(\begin{array}{l}
\psi_{\uparrow}(x, y) \\
\psi_{\downarrow}(x, y)
\end{array}\right) \\
= & \sum_{n}\left(\begin{array}{l}
\sum_{N} \tilde{t}_{n N \uparrow}^{\prime} e^{i k_{n \downarrow}^{+} y} \varphi_{n \uparrow}(x)+\sum_{n^{\prime}} \widetilde{r}_{n n^{\prime} \uparrow}^{\prime} e^{-i k_{n !}^{+}(L-y)} \varphi_{n \uparrow}(x) \\
\sum_{n N \downarrow}^{\prime} e^{i k_{n !}^{+} y} \varphi_{n \downarrow}(x)+\sum_{n^{\prime}} \tilde{r}_{n n^{\prime} \downarrow}^{\prime} e^{-i k_{n \downarrow}^{+}(L-y)} \varphi_{n \downarrow}(x)
\end{array}\right),
\end{aligned}
$$

and the local spin polarization $[12,14]$

$$
\left\langle S_{i}(x, y)\right\rangle=\frac{\hbar}{2} \Psi^{\dagger}(x, y) \sigma_{i} \Psi(x, y) .
$$

In the following, we present some numerical examples of the calculated spin polarization (in units of $\hbar=1$ ) in the QW at low temperatures. It should be pointed out that we 


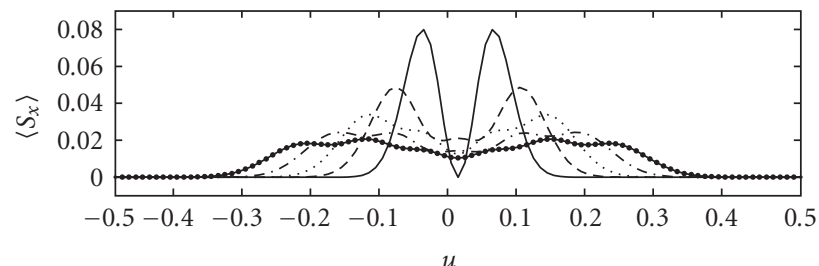

(a)

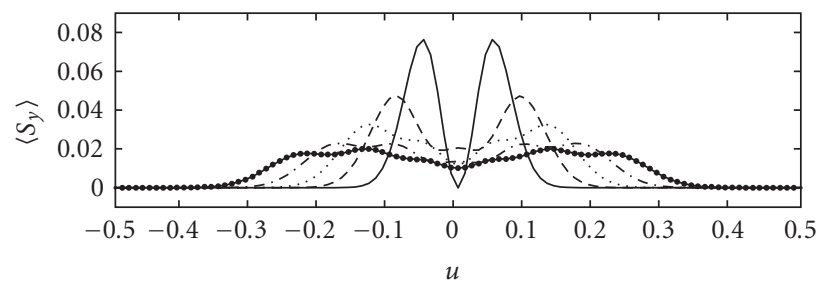

(b)

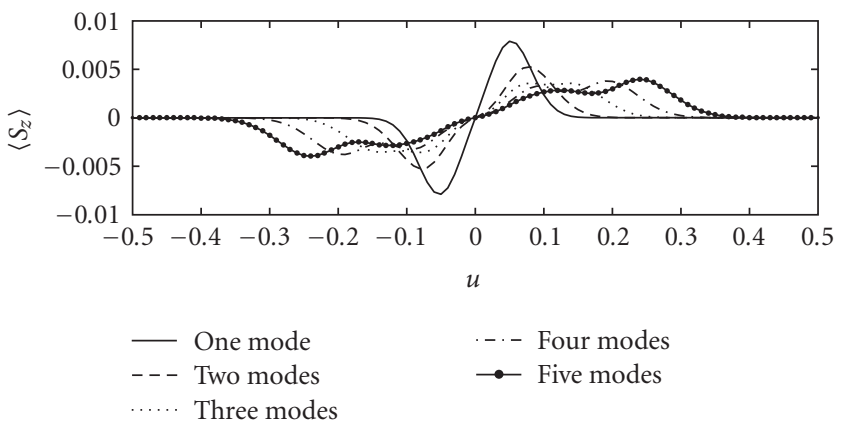

(c)

FIgURE 2: The spin polarization components $\left\langle S_{i}\right\rangle(i=x, y, z)$ as a function of the transverse position $u$. The Rashba strength $\alpha_{R}=0.5 \times 10^{-11} \mathrm{eVm}$ and $\left\langle S_{z}\right\rangle$ indicates the out-of-plane spin accumulation.

only consider the weak RSOC case [16-19], that is, $l_{\omega_{0}} / l_{\alpha_{R}}=$ $2 m^{*} \alpha_{R} / \hbar^{2} \alpha_{0}=0.1$ with $l_{\omega_{0}}=1 / \alpha_{0}=\left(\hbar / m^{*} \omega_{0}\right)^{1 / 2}$, and the width of two leads supporting number of propagating modes up to $N=6$. For the case of $\alpha_{R}=0.5 \times 10^{-11} \mathrm{eVm}$ [7], the incident electron energy is taken as $E=E_{\uparrow \downarrow}=7 \hbar \omega_{0}=$ $0.0367 \mathrm{eV}$ (for $\alpha_{R}=1.0 \times 10^{-11} \mathrm{eVm}, E=0.147 \mathrm{eV}$ ). Our system is a WNW QW $(n<N)$; however, the actual width of QW is chosen as $W=l_{\alpha_{R}}$ and the parabolic confining potential controls the number of propagation modes $n$ in the QW with the corresponding character oscillator frequency length $l_{\omega}<W$. The electron effective mass is taken as that for InGaAs quantum well $m^{*}=0.037 m_{e}[8]$ and the length of QW is chosen as $L=1200 \mathrm{~nm}$.

Figure 1 shows the 2D contour of spin polarization components $\left\langle S_{i}\right\rangle(i=x, y, z)$ for the cases of three and five propagation modes in the QW. It is noted that the components $\left\langle S_{x}\right\rangle$ and $\left\langle S_{y}\right\rangle$ present periodic oscillations along the longitudinal direction which comes from Rashba spin precession [15] while no oscillations presented for $\left\langle S_{z}\right\rangle$. This is the main difference from the results of [14] due to the different confining potential.

The polarization components $\left\langle S_{i}\right\rangle$ versus the transverse position $u=x / W$ for different propagation modes in the

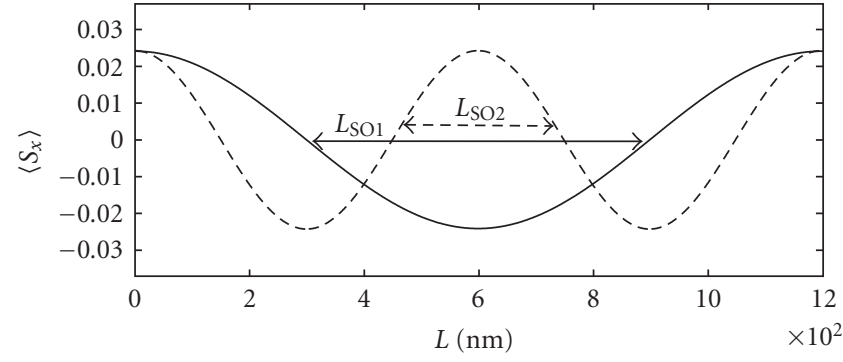

(a)

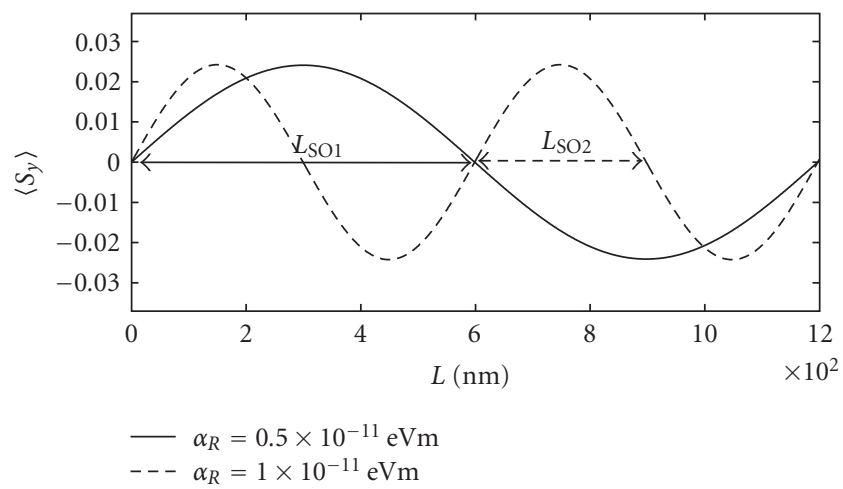

(b)

FIGURE 3: The intrinsic oscillations of spin polarization components $\left\langle S_{x}\right\rangle$ and $\left\langle S_{y}\right\rangle$ for $\alpha_{R}=0.5 \times 10^{-11} \mathrm{eVm}$ (dashed line) and $\alpha_{R}=$ $1.0 \times 10^{-11} \mathrm{eVm}$ (solid line) with $n=4$ and the transverse position $u=0.15$.

QW are demonstrated in Figure 2, with the longitudinal positions for $\left\langle S_{x}\right\rangle$ and $\left\langle S_{y / z}\right\rangle$ as 0 and $300 \mathrm{~nm}$, respectively. More peaks and extended polarization width toward two edges of the QW have been shown with the increasing modes $n$. The component $\left\langle S_{z}\right\rangle$ presents two inverse-direction peaks (shifting to the two edges of the QW), consequently, produces the out-of-plane spin accumulation in the QW. The spin accumulation is formed with the spin distribution polarized along the $z$ direction due to the interaction between the pseudomagnetic field arising from RSOC and the accelerating electrons $[20,21]$. Moreover, the net spin polarization rather than in-plane spin accumulation is illustrated for $\left\langle S_{x / y}\right\rangle$ while the present results of $\left\langle S_{z}\right\rangle$ are qualitatively consistent with $[12,14]$.

Furthermore, in Figure 3 we plot the longitudinal profile of spin polarization components $\left\langle S_{x}\right\rangle$ and $\left\langle S_{y}\right\rangle$ at a fixed transverse position $u=0.15$. As can be seen, due to the Rashba spin precession along the longitudinal position of $\mathrm{QW}$, the period of the oscillations (with $\pi / 2$ phase separation) for Rashba strength $\alpha_{R}=1.0 \times 10^{-11} \mathrm{eVm}$ is half that of the $0.5 \times 10^{-11} \mathrm{eVm}$ case. One notes that the Rashba precession lengths $L_{\mathrm{SO} 1}$ and $L_{\mathrm{SO} 2}$ are $600 \mathrm{~nm}$ and $300 \mathrm{~nm}$, respectively, which are consistent with the results $\left(L_{S O}=\pi \hbar^{2} /\left(2 m^{*} \alpha_{R}\right)\right)$ in $[14,22]$. It should be pointed out that the presented results may be observable experimentally to differentiate the intrinsic and extrinsic spin accumulation.

In conclusion, we have studied the spin polarization in a Rashba QW confined by a parabolic potential. It is found 
that the two inverse direction peaks of the out-of-plane spin accumulation $\left\langle S_{z}\right\rangle$ shift to the two edges of the QW with the increasing propagation modes. Furthermore, the intrinsic oscillation of spin polarization due to Rashba spin precession can be used to identify the intrinsic spin accumulation by changing the RSOC strength.

\section{Acknowledgments}

This work is supported by the Nature Science Foundation of China under Grant no. 10574042, the Specialized Research Fund for the Doctoral Program of Higher Education of China under Grant no. 20060542002, and the Hunan Provincial Natural Science Foundation of China under Grant no. 06JJ2097.

\section{References}

[1] G. A. Prinz, “Magnetoelectronics," Science, vol. 282, no. 5394, pp. 1660-1663, 1998.

[2] P. Ball, "Meet the spin doctors...," Nature, vol. 404, no. 6781, pp. 918-920, 2000.

[3] I. Žutić, J. Fabian, and S. Das Sarma, "Spintronics: fundamentals and applications," Reviews of Modern Physics, vol. 76, no. 2, pp. 323-410, 2004.

[4] J. Nitta, T. Akazaki, H. Takayanagi, and T. Enoki, "Gate control of spin-orbit interaction in an inverted $\mathrm{In}_{0.53} \mathrm{Ga}_{0.47} \mathrm{As} / \mathrm{In}_{0.52} \mathrm{Al}_{0.48}$ As heterostructure," Physical Review Letters, vol. 78, no. 7, pp. 1335-1338, 1997.

[5] Z. Li and Z. Yang, "Influence of evanescent waves on spinpolarization in a ballistic Rashba bar," Physical Review B, vol. 76, no. 3, Article ID 033307, 4 pages, 2007.

[6] Z. Li and Z. Yang, "Effects of extended and localized states on spin Hall polarization in ballistic Rashba structures," Physical Review B, vol. 77, no. 20, Article ID 205322, 4 pages, 2008.

[7] S. Bellucci and P. Onorato, "Spin filtering and spin Hall accumulation in an interferometric ballistic nanojunction with Rashba spin-orbit interaction," Physical Review B, vol. 77, no. 7, Article ID 075303, 9 pages, 2008.

[8] F. Zhai and H. Q. Xu, "Spin filtering and spin accumulation in an electron stub waveguide with spin-orbit interaction," Physical Review B, vol. 76, no. 3, Article ID 035306, 5 pages, 2007.

[9] H.-F. Lü and Y. Guo, "Kondo effect and spin-polarized transport through a quantum dot with Rashba spin-orbit interaction," Physical Review B, vol. 76, no. 4, Article ID 045120, 9 pages, 2007.

[10] Y. K. Kato, R. C. Myers, A. C. Gossard, and D. D. Awschalom, "Observation of the spin Hall effect in semiconductors," Science, vol. 306, no. 5703, pp. 1910-1913, 2004.

[11] A. D. Giddings, M. N. Khalid, T. Jungwirth, et al., "Large tunneling anisotropic magnetoresistance in (Ga,Mn)As nanoconstrictions," Physical Review Letters, vol. 94, no. 12, Article ID 127202, 4 pages, 2005.

[12] Q. Wang and L. Sheng, "Current induced local spin polarization due to the spin-orbit coupling in a two dimensional narrow strip," International Journal of Modern Physics B, vol. 19, no. 27, pp. 4135-4142, 2005.

[13] B. K. Nikolić, S. Souma, L. P. Zârbo, and J. Sinova, "Nonequilibrium spin Hall accumulation in ballistic semiconductor nanostructures," Physical Review Letters, vol. 95, no. 4, Article ID 046601, 4 pages, 2005.
[14] J. Yao and Z. Q. Yang, "Spin accumulation in a ballistic Rashba bar," Physical Review B, vol. 73, no. 3, Article ID 033314, 4 pages, 2006.

[15] J. Wang, K. S. Chan, and D. Y. Xing, "Intrinsic oscillation of spin accumulation induced by Rashba spin-orbital interaction," Physical Review B, vol. 73, no. 3, Article ID 033316, 4 pages, 2006.

[16] A. V. Moroz and C. H. W. Barnes, "Effect of the spin-orbit interaction on the band structure and conductance of quasione-dimensional systems," Physical Review B, vol. 60, no. 20, pp. 14272-14285, 1999.

[17] F. Kassubek, C. A. Stafford, and H. Grabert, "Force, charge, and conductance of an ideal metallic nanowire," Physical Review B, vol. 59, no. 11, pp. 7560-7574, 1999.

[18] G. Liu and G. Zhou, "A possible realization of spin filter using a quantum wire with Rashba spin-orbit coupling," Journal of Applied Physics, vol. 101, no. 6, Article ID 063704, 4 pages, 2007.

[19] G. Liu and G. Zhou, "Conductance for a quantum wire with weak Rashba spin-orbit coupling," Chinese Physics Letters, vol. 22, no. 12, pp. 3159-3162, 2005.

[20] S.-Q. Shen, "Spin Hall effect and Berry phase in twodimensional electron gas," Physical Review B, vol. 70, no. 8, Article ID 081311, 4 pages, 2004.

[21] J. Sinova, D. Culcer, Q. Niu, N. A. Sinitsyn, T. Jungwirth, and A. H. MacDonald, "Universal intrinsic spin Hall effect," Physical Review Letters, vol. 92, no. 12, Article ID 126603, 4 pages, 2004.

[22] B. K. Nikolić and S. Souma, "Decoherence of transported spin in multichannel spin-orbit-coupled spintronic devices: scattering approach to spin-density matrix from the ballistic to the localized regime," Physical Review B, vol. 71, no. 19, Article ID 195328, 15 pages, 2005. 

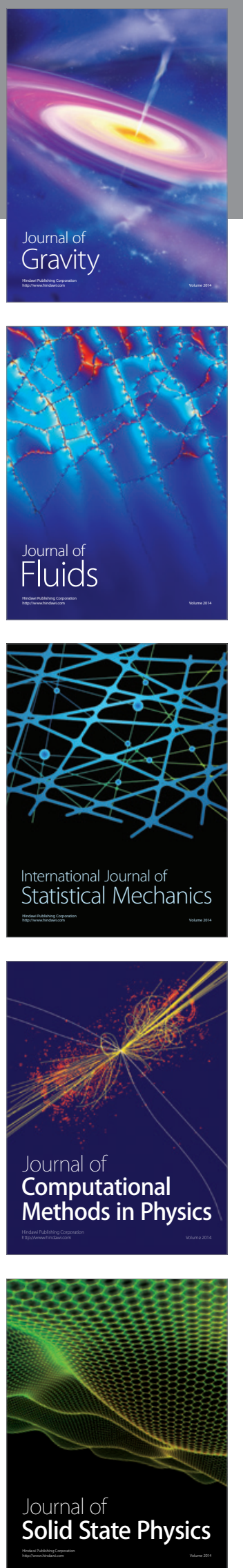

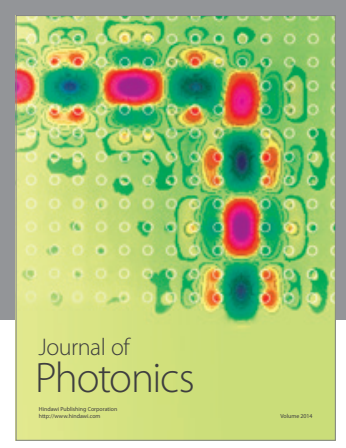

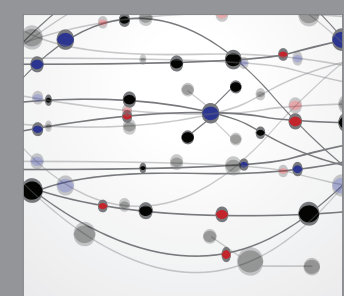

The Scientific World Journal
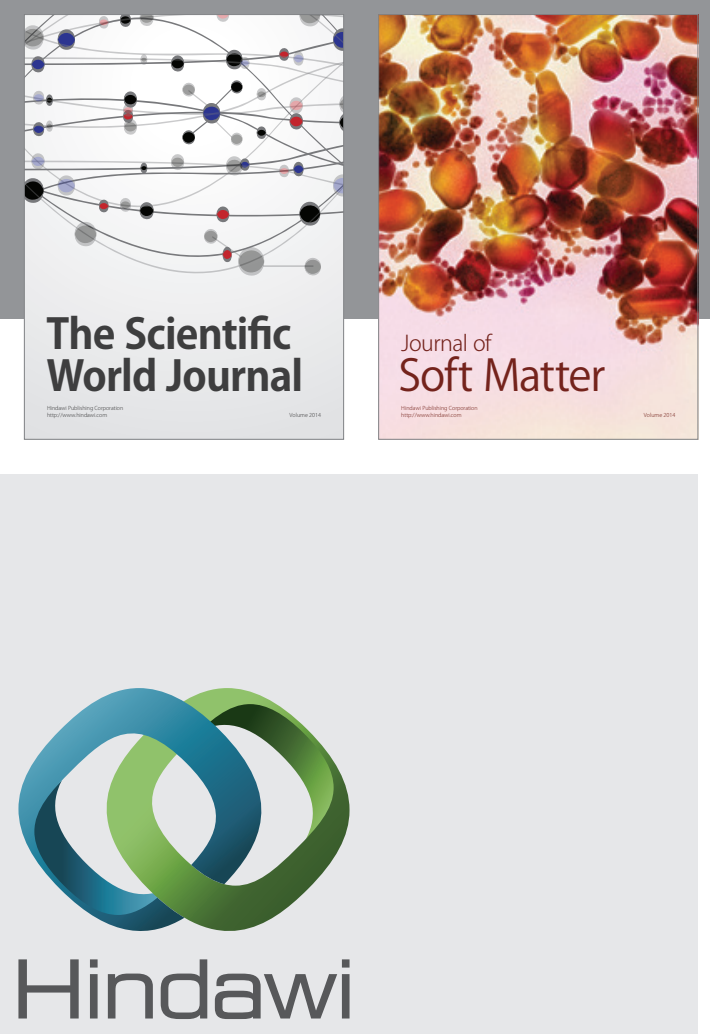

Submit your manuscripts at

http://www.hindawi.com
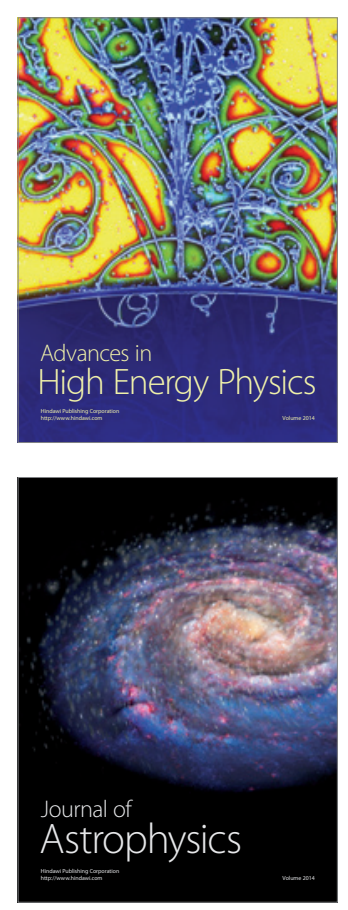
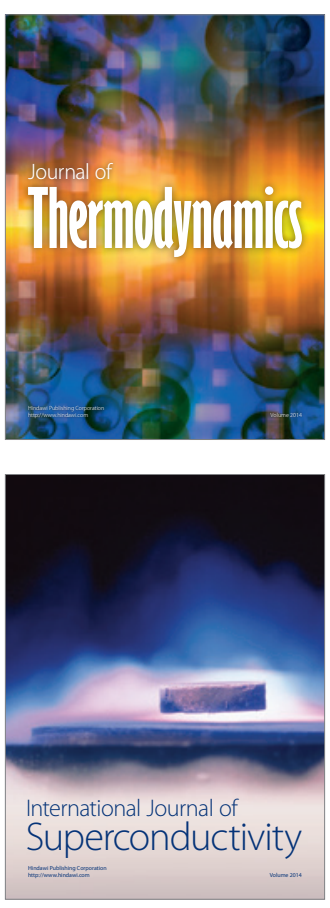
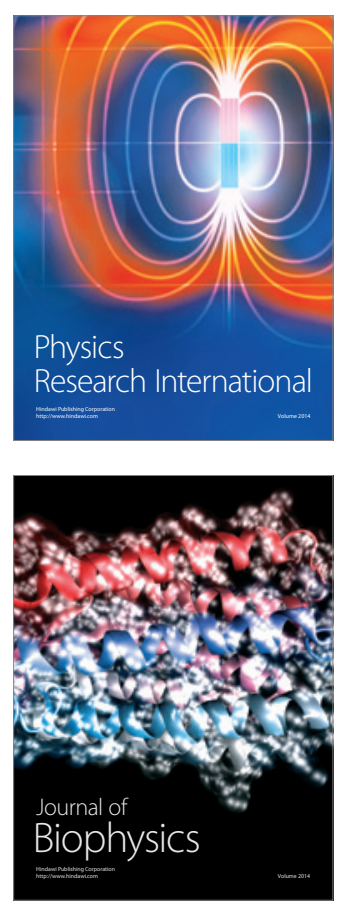
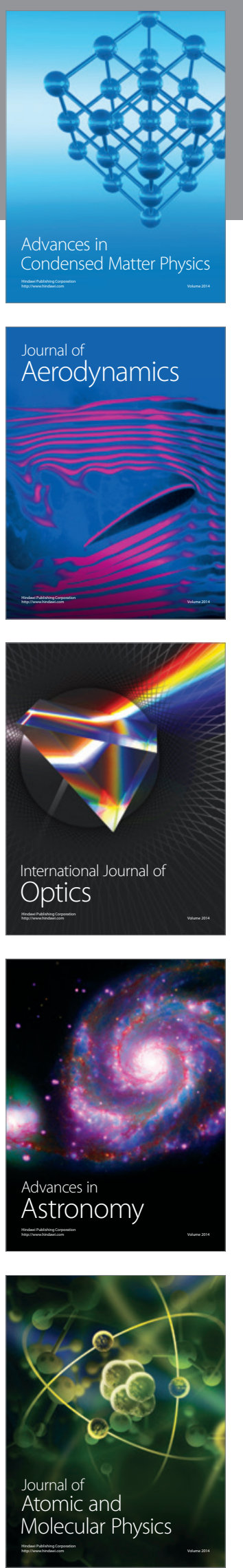